\title{
Flexural Vibration Test of a Beam Elastically Restrained at One End: A New Approach for Young's Modulus Determination
}

\author{
Rafael M. Digilov and Haim Abramovich \\ Faculty of Aerospace Engineering, Technion-Israel Institute of Technology, 32000 Haifa, Israel \\ Correspondence should be addressed to Rafael M. Digilov; edurafi@technion.ac.il
}

Received 1 May 2013; Accepted 11 July 2013

Academic Editor: Xing Chen

Copyright (c) 2013 R. M. Digilov and H. Abramovich. This is an open access article distributed under the Creative Commons Attribution License, which permits unrestricted use, distribution, and reproduction in any medium, provided the original work is properly cited.

\begin{abstract}
A new vibration beam technique for the fast determination of the dynamic Young modulus is developed. The method is based on measuring the resonant frequency of flexural vibrations of a partially restrained rectangular beam. The strip-shaped specimen fixed at one end to a force sensor and free at the other forms the Euler Bernoulli cantilever beam with linear and torsion spring on the fixed end. The beam is subjected to free bending vibrations by simply releasing it from a flexural position and its dynamic response detected by the force sensor is processed by FFT analysis. Identified natural frequencies are initially used in the frequency equation to find the corresponding modal numbers and then to calculate the Young modulus. The validity of the procedure was tested on a number of industrial materials by comparing the measured modulus with known values from the literature and good agreement was found.
\end{abstract}

\section{Introduction}

The Young modulus is a fundamental material property and its determination is common in science and engineering $[1,2]$. It is a key parameter in mechanical engineering design to predict the behavior of the material under deformation forces or more to get an idea of the quality of the material. Young's moduli are determined from static and dynamic tests. In static measurements $[3,4]$ such as the classical tensile or compressive test, a uniaxial stress is exerted on the material, and the elastic modulus is calculated from the transverse and axial deformations as the slope of the stress-strain curve at the origin. Dynamic methods [5-12] are more precise and versatile since they use very small strains, far below the elastic limit and therefore are virtually nondestructive allowing repeated testing of the same sample. These include the ultrasonic pulse-echo $[6,7]$ or bar resonance methods $[4,8-14]$. In the sonic pulse technique, the dynamic Young modulus is determined by measuring the sound velocity in the sample. In the resonance method, the linear elastic, uniform, and isotropic material of density $\rho$ usually in the form of a bar of known dimensions is subjected to transverse or flexural vibrations, the natural frequency of $n$th mode of which $f_{n}$ related to Young's modulus $E$ by the relation $[15,16]$

$$
f_{n}=\frac{\lambda_{n}^{2}}{2 \pi l^{2}} \sqrt{\frac{E I}{\rho A}}, \quad n=1,2,3, \ldots
$$

can be accurately measured. In (1) $\lambda_{n}$ is the modal eigenvalue that depends on boundary conditions, $l$ is the vibrating length of the bar, $A$ is its cross-sectional area, and $I$ is the second moment of the cross-section, equal to $\pi r^{4} / 4$ for a rod of radius $r$ and $d h^{3} / 12$ for a rectangular beam with width $d$ and depth $h$. Knowing the modal numbers, by simply measuring the resonance frequencies, geometry, and density of the specimen, the Young modulus can be determined from (1) as

$$
E=\frac{4 \pi^{2} f_{n}^{2} l^{4} \rho A}{\lambda_{n}^{4} I} .
$$

The test sample is usually arranged in a manner to simulate free-free or clamped-free end conditions [10-12], when $\lambda_{n}$, associated with the $n$th flexural mode is a constant. 


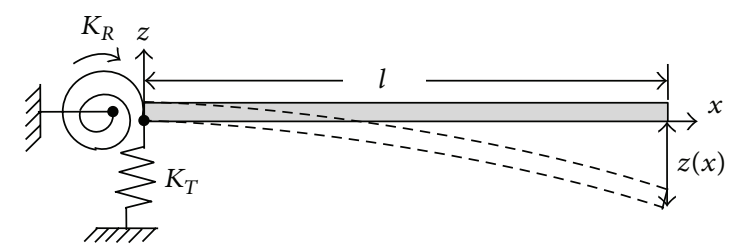

FIGURE 1: Model of a cantilever beam elastically restrained at $x=0$.

In the present paper, we develop a new approach, in which a rectangular strip-shaped sample attached to a force sensor forms the Euler Bernoulli beam with partial translational and rotational restraints at the fixed end. This feature expands the capabilities of the resonant beam method making it suitable for materials with high stiffness and low density in which case, it is difficult to ascertain the flexural resonance frequencies with high certainty.

\section{Theoretical Background}

Consider a rectangular bar of uniform density $\rho$, crosssection dimensions of which width $d$ and depth $h$ are much less than length $l$. The bar is fixed at one end $(x=0)$ to the force sensor with a linear $\left(K_{T}\right)$ and torsion $\left(K_{R}\right)$ springs constants (see Figure 1) and is otherwise free to move in the transverse $z$-direction. For small deflections, that is, $\partial z(x, t) / \partial x \ll 1$, the effects of rotary inertia and shear deformation can be ignored. In this case, neglecting the deflection due to the weight, the flexural displacement of a bar, $z(x, t)$ at point $x$ is governed by the Euler-Bernoulli equation $[10]$

$$
\rho A \frac{\partial^{2} z}{\partial t^{2}}+E I \frac{\partial^{4} z}{\partial x^{4}}=0
$$

with boundary conditions at $x=0$ :

$$
-K_{T} z(0, t)=\left.E I \frac{\partial^{3} z}{\partial x^{3}}\right|_{x=0},\left.\quad K_{R} \frac{\partial z}{\partial x}\right|_{x=0}=\left.E I \frac{\partial^{2} z}{\partial x^{2}}\right|_{x=0}
$$

which correspond to the force and moment balance, respectively. At the free end $x=l$ there are no moment and shear force acting, that is,

$$
\left.\frac{\partial^{2} z}{\partial x^{2}}\right|_{x=l}=0,\left.\quad \frac{\partial^{3} z}{\partial x^{3}}\right|_{x=l}=0
$$

Equations (3)-(5) define completely the linear flexural vibration problem, in which the natural frequencies of the beam depend on spring constants $K_{T}$ and $K_{R}$. Applying the separation of variables method, the solution of (3) can be cast in the following form:

$$
z(x, t)=w(x) T(t)=\sum_{n=1}^{\infty} w_{n}(x) e^{i \omega_{n} t}
$$

where $w_{n}(x)$ describes the $n$th normal mode and $\omega_{n}$ $\left(=2 \pi f_{n}, f\right.$ : resonant frequency) is the angular frequency of the $n$th mode. Substituting (6) into (3) gives an eigenvalue problem in the form of a fourth-order ordinary differential equation

$$
\frac{\partial^{4} w_{n}(x)}{\partial x^{4}}-\kappa_{n}^{4} w_{n}(x)=0,
$$

where $\kappa_{n}$ is related to the angular frequency $\omega_{n}$ and the modal number $\lambda_{n}$ by the dispersion relationship

$$
\kappa_{n}^{4}=\left(\frac{\lambda_{n}}{l}\right)^{4}=\omega_{n}^{2} \frac{\rho A}{E I} .
$$

With the boundary condition, (4), the solution of (7) admits the form of $n$th flexural eigenmode:

$$
\begin{aligned}
w_{n}(x)= & A_{n}\left(\cosh \kappa_{n} x+a_{n} \sinh \kappa_{n} x+b_{n} \sin \kappa_{n} x\right) \\
& +C_{n}\left(\cos \kappa_{n} x-b_{n} \sinh \kappa_{n} x-a_{n} \sin \kappa_{n} x\right),
\end{aligned}
$$

where related coefficients $b_{n}$ and $a_{n}$ are defined as

$$
a_{n}=\frac{1-\lambda_{n}^{4} R_{n} T_{n}}{2 \lambda_{n}^{3} R_{n}}, \quad b_{n}=\frac{1+\lambda_{n}^{4} R_{n} T_{n}}{2 \lambda_{n}^{3} R_{n}}
$$

with $R_{n}$ and $T_{n}$ expressed through experimentally accessible quantities:

$$
R_{n}=\frac{K_{R}}{\left(2 \pi f_{n}\right)^{2} \rho A l^{3}}, \quad T_{n}=\frac{K_{T}}{\left(2 \pi f_{n}\right)^{2} \rho A l},
$$

and integration constants $A_{n}$ and $C_{n}$ are related by the modal restriction equation (5) as

$$
\begin{aligned}
\frac{A_{n}}{C_{n}} & =\frac{\cos \lambda_{n}+b_{n} \sinh \lambda_{n}-a_{n} \sin \lambda_{n}}{\cosh \lambda_{n}+a_{n} \sinh \lambda_{n}-b_{n} \sin \lambda_{n}} \\
& =-\frac{\sin \lambda_{n}-b_{n} \cosh \lambda_{n}+a_{n} \cos \lambda_{n}}{\sinh \lambda_{n}+a_{n} \cosh \lambda_{n}-b_{n} \cos \lambda_{n}},
\end{aligned}
$$

where the second equality implies the constraints on possible values of $\lambda_{n}$, known as the frequency equation:

$$
\begin{aligned}
\left(1+\lambda_{n}^{4} R_{n} T_{n}\right) & -\left(1-\lambda_{n}^{4} R_{n} T_{n}\right) \cos \lambda_{n} \cosh \lambda_{n} \\
-\lambda_{n}[ & \left(\lambda_{n}^{2} R_{n}+T_{n}\right) \sin \lambda_{n} \cosh \lambda_{n} \\
& \left.+\left(\lambda_{n}^{2} R_{n}-T_{n}\right) \sinh \lambda_{n} \cos \lambda_{n}\right]
\end{aligned}
$$

$=0$.

For given values of $R_{n}$ and $T_{n}$, transcendental equation (13) has infinite (iterative or graphical) solutions. As $T_{n} \rightarrow 0$ and $R_{n} \rightarrow 0$ (13) becomes

$$
1-\cos \lambda_{n} \cosh \lambda_{n}=0
$$

that is the frequency equation for the free-free beam. For $T_{n} \gg R_{n}$ (13) reduces to the frequency equation derived by Chun [17], which in our case is written in the form

$$
\begin{aligned}
R_{n} \lambda_{n}^{3} & \left(1+\cos \lambda_{n} \cosh \lambda_{n}\right) \\
& -\sin \lambda_{n} \cosh \lambda_{n}-\sinh \lambda_{n} \cos \lambda_{n}=0
\end{aligned}
$$

which, in turn, at $R_{n} \rightarrow \infty$ becomes the frequency equation for the clamped-free beam

$$
1+\cos \lambda_{n} \cosh \lambda_{n}=0 .
$$




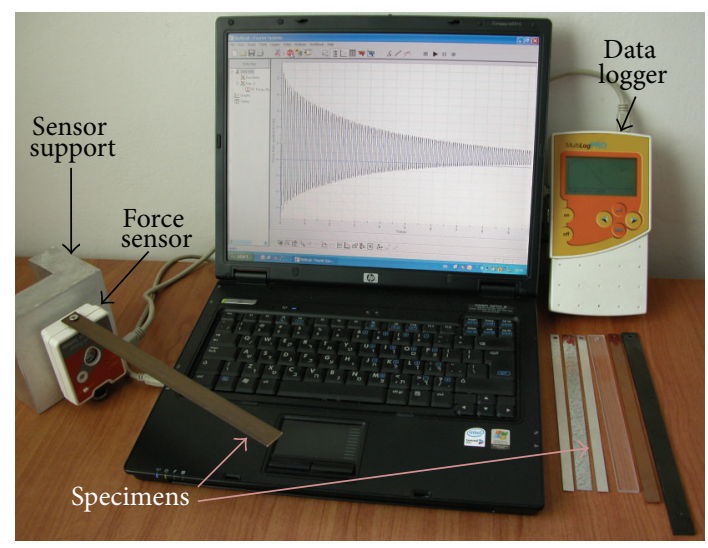

FIGURE 2: Equipment used for measuring Young's modulus with the force sensor.

\section{Principles of Operation}

The experimental setup consists of a commercial, Fourier Force Sensor DT 272 with rotation $K_{R}(=17.837 \mathrm{~N} \cdot \mathrm{m})$ and translation $K_{T}\left(=6400 \mathrm{~N} \cdot \mathrm{m}^{-1}\right)$ spring constants, accuracy $\pm 2 \%$, and resolution (12 bit) $0.005 \mathrm{~N}$ for a scale range $\pm 10 \mathrm{~N}$. The force sensor mounted on a support is connected through data acquisition system and data studio software to a personal computer (PC) as is shown in Figure 2.

The strip-shaped specimen with a roughly mass of $m \approx$ $10 \mathrm{~g}$ is attached horizontally to a force sensor at one end and is free at the other end. By simply displacing the free end in the transverse direction and abruptly releasing it, the sample is subjected to free flexural vibrations, so that the condition $\partial z / \partial x \ll 1$ was fulfilled. At a given sample rate, $f_{s}=1000 \mathrm{~Hz}$ the force sensor detects the dynamic response and through the data acquisition system displays the damped oscillations of the restoring force versus time (Figure 3(a)). This vibration signal is analyzed and processed by an FFT implemented in acquisition software like MultiLogPRO [18] providing the direct information about natural frequencies up to $f_{s} / 2$, which appears as single peaks in the frequency spectrum (Figure 3(b)). At this point, using data on the geometrical dimensions of the sample, its density, and spring constants of the force sensor $K_{R}$ and $K_{T}$, identified resonance frequencies $f_{n}$ are used in (11) to calculate dimensionless parameters $R_{n}$ and $T_{n}$. Knowing $R_{n}$ and $T_{n}$, modal number $\lambda_{n}$ was found from the graphical solution of (13) using the mathematical packages MATHEMATICA [19]. The Young modulus is then determined from

$$
E=48 \pi^{2} \frac{\rho f_{n}^{2} l^{4}}{\lambda_{n}^{4} h^{2}} .
$$

The relative error of the method arises from the uncertainties in the measurement of the quantities in (17). The relative error in the density $\rho$ determined by the hydrostatic method is $|d(\ln \rho)| \leq 0.7 \%$. The uncertainty in the thickness and length measurement are, respectively, $|d(\ln h)| \leq 0.4 \%$ and $|d(\ln l)| \leq 0.25 \%$. The dispersion in the natural frequency
TABLE 1: Identified resonant frequencies $f_{n}$ in $\mathrm{Hz}$, modal numbers determined from (13), and Young's moduli in GPa evaluated by (17) for the brass specimens of different lengths, $d=16 \mathrm{~mm}, h=1.5 \mathrm{~mm}$, and $\rho=8400 \mathrm{~kg} \cdot \mathrm{m}^{-3}$.

\begin{tabular}{lccccccc}
\hline$l / d$ & $T_{n} / R_{n}$ & $f_{1}$ & $\lambda_{1}$ & $E_{1}$ & $f_{2}$ & $\lambda_{2}$ & $E_{2}$ \\
\hline 8.13 & 60.6 & 37.82 & 1.595 & 111.6 & 232.79 & 4.068 & 100 \\
9.06 & 75.4 & 31.14 & 1.619 & 110.3 & 193.83 & 4.14 & 100 \\
9.813 & 88.4 & 26.84 & 1.638 & 107.4 & 170.23 & 4.179 & 102 \\
10.69 & 104.9 & 23.07 & 1.653 & 107.9 & 147.82 & 4.215 & 104.7 \\
12.25 & 137.8 & 17.9 & 1.679 & 105.2 & 115.24 & 4.276 & 103.7 \\
14.69 & 198.2 & 13.0 & 1.701 & 108.9 & 84.10 & 4.326 & 109 \\
15.5 & 220.7 & 11.75 & 1.709 & 108.2 & 76.25 & 4.372 & 106.5 \\
\hline
\end{tabular}

determination is $\left|d\left(\ln f_{1}\right)\right| \leq 0.25 \%$. By applying the error propagation technique, given by

$$
|d(\ln E)| \leq 2\left|d\left(\ln f_{1}\right)\right|+|d(\ln \rho)|+4|d(\ln l)|+2|d(\ln h)|
$$

we find that the relative error in Young's modulus does not exceed $\pm 3 \%$. The greatest inaccuracies occurred in the measurement of the spacemen dimensions.

\section{Results and Discussion}

To test the accuracy and validity of the present method, the effect of the sample length on the resonance frequency and Young's modulus was studied. A commercial brass strip of width $d=16 \mathrm{~mm}$, thickness $h=1.5 \mathrm{~mm}$, and density $\rho=$ $8400 \mathrm{~kg} \cdot \mathrm{m}^{-3}$ was cut into samples of various length, so that one of the conditions of the Euler-Bernoulli beam theory $l / h>10$ remained unchanged, while the second $l / d$ ranged from 8 to 16 . Since the ration $T_{n} / R_{n} \gg 1$ (see Table 1) we used the approximated equation (15) to find the $\lambda_{1}$. The validity of this approach is illustrated in Figure 4, which shows that the solutions of both (15) and (13) practically coincide.

Based on the results of measurements of the natural frequencies and modal numbers presented in Table 1, a double logarithm plot of $f_{i} / \lambda_{i}^{2}$ against $l$ predicted by (1) to be linear with the gradient of -2 shows that the slope of the line that best fits these data in a least-squares sense is -2.02 for the first mode and -1.94 for the second one. Based on the same set of data we show in Figure 5 the plot of the ratio $f_{i} l^{2} / \lambda_{i}^{2}$ versus $l / d$. Most of the uncertainty in the ordinates of this plot arises from uncertainty of $l$ rather than $f_{i}$. In the range of $l / d>10$, the value of $f_{i} l^{2} / \lambda_{i}^{2}$ is constant to within the experimental uncertainties, showing that $f_{i} / \lambda_{i}^{2} \propto l^{-2}$ is in agreement with (1). For $l / d<10$, that is smaller for validity of the Euler-Bernoulli beam theory, the ratio $f_{i} l^{2} / \lambda_{i}^{2}$ decreases (increases) with $l$ for the first (second) mode. For $l / d \geq 10$, the mean value of Young's modulus $E=106 \pm 2 \mathrm{GPa}$ lies within the range $95 \div 110 \mathrm{GPa}$, listed in an extensive table of ASTM testing [20].

Below, we compare the results of measurements of elastic moduli at ambient temperature for a wide class of industrial materials with that accepted in the literature. A set of test specimens used were cut from the commercial sheet materials 


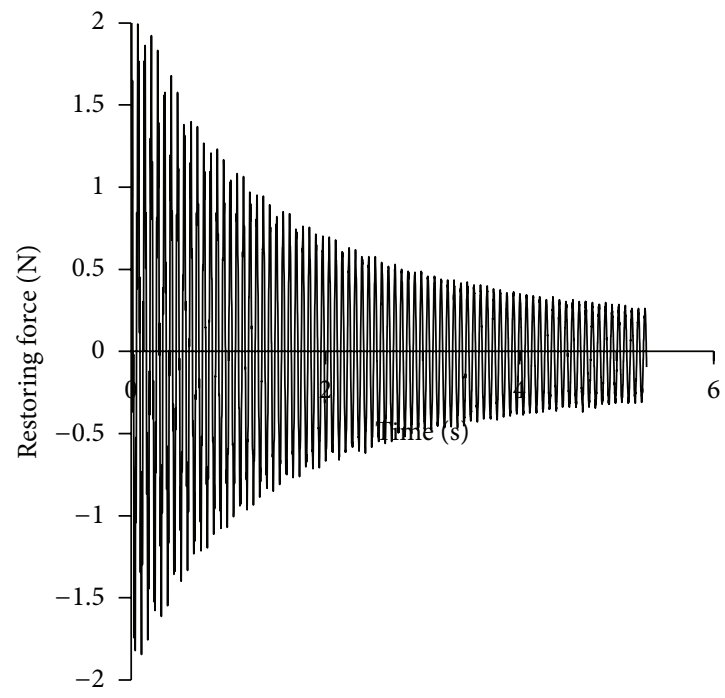

(a)

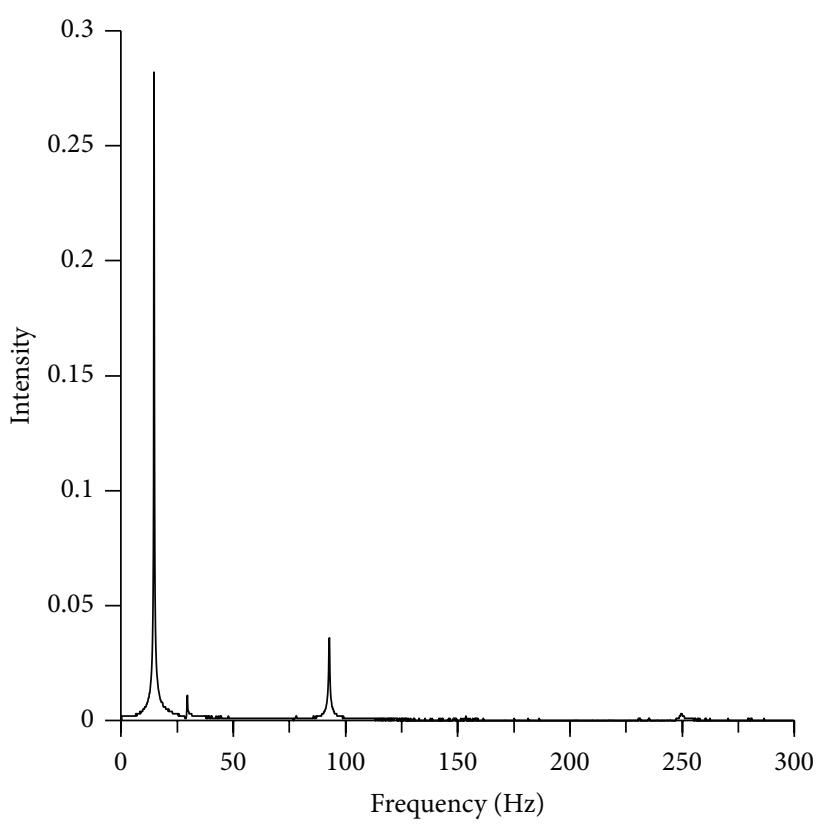

(b)

Figure 3: The flexural vibration test of partially restrained aluminum beam with $l=0.282 \mathrm{~m}, d=1.35 \mathrm{~cm}$, and $h=1.55 \mathrm{~mm}$. (a) Dynamic response detected by the force sensor at sample rate $1 \mathrm{kHz}$. (b) Identified resonance frequencies: $f_{1}=14.7 \mathrm{~Hz} ; f_{2}=92.8 \mathrm{~Hz} ; f_{3}=249.5 \mathrm{~Hz}$.

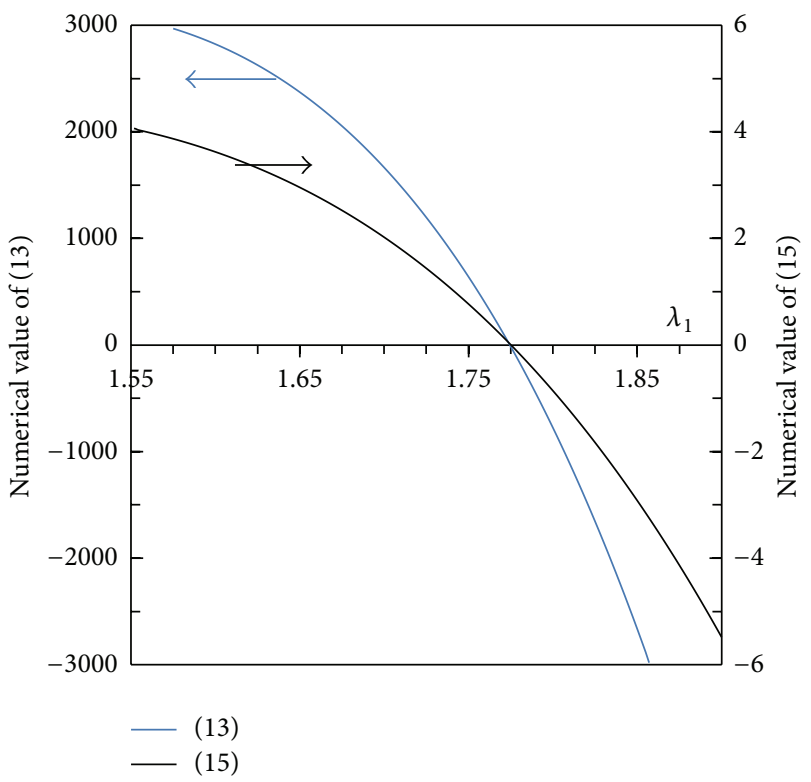

FIGURE 4: Graphical solutions of (13) and (15) for the first mode of flexural vibrations of partially restrained aluminum beam are, respectively, $\lambda_{1}=1.7752$ and $\lambda_{1}=1.7745$ against $\lambda_{1}=1.8751$ for ideal clamped-free case.

into strips of the thickness $(h)$ from 0.5 to $3.3 \mathrm{~mm}$, width (d) from 5 to $16.5 \mathrm{~mm}$, and length (l) from 15 to $30 \mathrm{~cm}$. For each specimen, the length, width, and thickness were altered and the value of the Young modulus calculated by (17) for each set varied within the experimental error. Table 2 summarizes data of the specimen dimension, material density, natural frequencies, modal numbers, and Young's modulus calculated from the first resonant frequency. It can be seen that test results are in good agreement with the accepted those in the literature data.

In all cases, identified natural frequencies of the partially restrained cantilever beam are lower than those for the clamped-free case. However, this does not necessary mean that the elastic modulus determined from these frequencies should be smaller than in ideal clamped-free case as the modal number, being in the denominator equation (17) in fourth power, decreases as well. Interestingly, the plot of $\lambda_{1}^{4}$ versus $1 / R_{1}$ in Figure 5 shows a linear dependence

$$
\lambda_{1}^{4}=\lambda_{\infty}^{4}\left(1-\frac{1}{3 R_{1}}\right)
$$

where $\lambda_{\infty}=1.8751$ is the first modal number for the clamped-free flexural vibrations of the beam. Taking into account (11), after substituting (19) in (17), we obtain the working equation for the Young modulus determination through the first resonance frequency of flexural vibrations of the partially restrained cantilever beam (Figure 6)

$$
E=\frac{48 \pi^{2} f_{1}^{2} \rho l^{4}}{h^{2} \lambda_{\infty}^{4}\left(1-\left(4 \pi^{2} f_{1}^{2} \rho A l^{3} / 3 K_{R}\right)\right)} .
$$

\section{Summary}

A new technique for the fast determination of the dynamic Young modulus was developed, yielding a substantial modification of the classical cantilever beam method. The procedure 
TABLE 2: Young's moduli of materials $E$ in GPa, determined at room temperature by (17) and specimen parameters: length $l$ in mm, thickness $h$ in $\mathrm{mm}$, density $\rho$ in $\mathrm{kg} \cdot \mathrm{m}^{-3}$, fundamental frequency $f_{1}$ in $\mathrm{Hz}$, and modal number $\lambda_{1}$ calculated by (13).

\begin{tabular}{lccccccc}
\hline Material & $l$ & $h$ & $\rho$ & $f_{1}$ & $\lambda_{1}$ & $E$ & $E_{\text {Literature }}$ \\
\hline Al 6061 sheet & 282 & 1.55 & 2715 & 14.42 & 1.778 & 70.0 & $70-72$ \\
Zn coated steel & 193 & 0.55 & 7820 & 12.0 & 1.868 & 201 & 206 \\
Sheet steel 304 & 193 & 0.5 & 7970 & 11.0 & 1.864 & 210 & $190-213$ \\
Cooper alloy & 214 & 1.0 & 8790 & 11.89 & 1.848 & 106 & $110-120$ \\
Brass stripe & 248 & 1.5 & 8400 & 11.75 & 1.709 & 108 & $96-110$ \\
Perspex & 190 & 3.0 & 1190 & 23.2 & 1.798 & 1.6 & $2.4-4.6$ \\
Wood, oak & 252 & 3.5 & 674 & 30.3 & 1.664 & 12.6 \\
Composite $^{\text {a }}$ & 232 & 2.5 & 1600 & 33.0 & 1.429 & 92.0 & $36-150$ \\
\hline
\end{tabular}

araphite carbon epoxy.

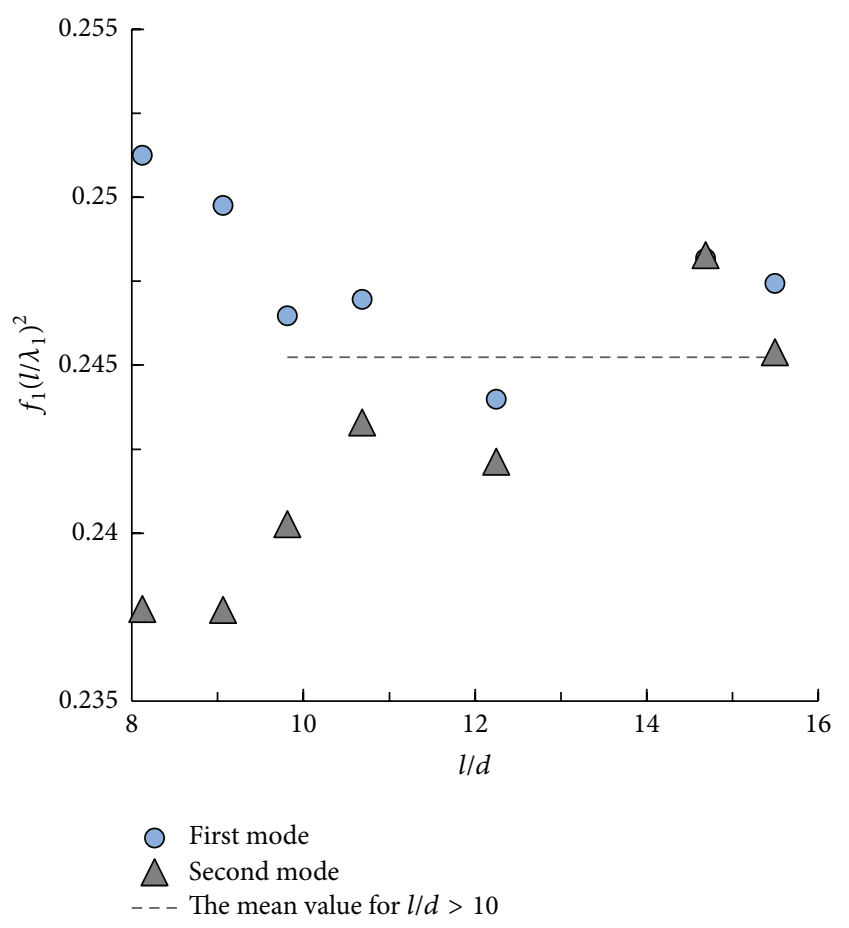

FIGURE 5: Plot of $f_{i}\left(l / \lambda_{i}\right)^{2}$ versus $l / d$ based on the flexural vibration test of a rectangular brass strip $(d=16.0 \mathrm{~mm}$ and $h=1.5 \mathrm{~mm})$. For $l / d \geq 10$, the mean value 0.245 (broken line) is constant to within experimental uncertainty.

uses a rectangular beam, partially restrained at one end, flexural vibrations of which are detected with the aid of the force sensor. The relative experimental uncertainty is found to be less than $3 \%$, which is mainly due to the uncertainty in the samples dimensions. The feasibility and accuracy of a new experimental procedure has been demonstrated by measuring the Young modulus for a number of test materials with different material properties. Comparison of obtained results with those accepted in the literature data is good. The relative deviation of measured values from the cited data is less than $5 \%$. The method has potential advantages over other dynamic methods of being very simple and fast and requiring no additional equipment to excite resonance frequencies. It is particularly suitable for composite materials having a high

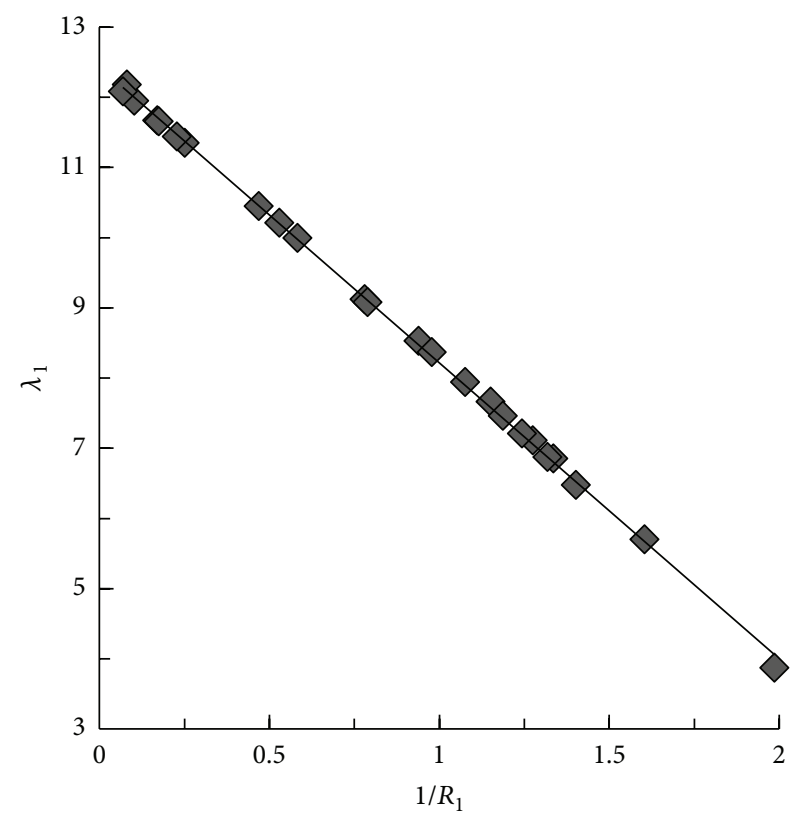

FIGURE 6: The modal number of the first mode of flexural vibrations of partially restrained cantilever beams in the fourth degree as a function of the inverse of the dimensionless frequency parameter, $1 / R_{1}$. Symbols are experimental data taken from Tables 1 and 2 . Solid line is by (19).

stiffness and low density, such as carbon fiber reinforced plastic. The accuracy can be significantly improved by more precise determination of specimen dimensions.

\section{Acknowledgment}

This work was supported by the Ministry of Absorption and Immigration of Israel through the KAMEA Science Foundation.

\section{References}

[1] J. D. Lord and R. Morrell, “"Elastic modulus measurement”. Measurement good practice guide, No. 98," Febraury 2007, http://www.npl.co.uk/publications/guides/guides-by-title/. 
[2] J. D. Lord, Review of Methods and Analysis Software for the Determination of Modulus from Tensile Tests, vol. 41, NPL Measurement Note MATC (MN), 2002.

[3] P. E. Armstrong, Measurement of Mechanical Properties, Techniques of Metals Research, vol. 5, part 2 of Edited by R. F. Bunshan, John Wiley \& Sons, New York, NY, USA, 1971.

[4] M. Radovic, E. Lara-Curzio, and L. Riester, "Comparison of different experimental techniques for determination of elastic properties of solids," Materials Science and Engineering A, vol. 368, no. 1-2, pp. 56-70, 2004.

[5] A. Wolfenden, Ed., Dynamic Elastic Modulus Measurements in Materials, American Society for Testing and Materials, 1990.

[6] A. S. Birks and R. E. Green, Nondestructive Testing Handbook, vol. 7 of Ultrasonic Testing, American Society for Nondestructive Testing, 1991.

[7] A. Migliori and J. L. Sarrao, Resonant Ultrasound Spectroscopy: Applications to Physics, Materials Measurements and Nondestructive Evaluation, John Wiley \& Sons, New York, NY, USA, 1997.

[8] A. Wolfenden, M. R. Harmouche, G. V. Blessing et al., "Dynamic Young's modulus measurements in metallic materials: results of an interlaboratory testing programm," Journal of Testing and Evaluation, 1989.

[9] G. Roebben, B. Bollen, A. Brebels, J. van Humbeeck, and O. van der Biest, "Impulse excitation apparatus to measure resonant frequencies, elastic moduli, and internal friction at room and high temperature," Review of Scientific Instruments, vol. 68, no. 12, pp. 4511-4515, 1997.

[10] W. Lins, G. Kaindl, H. Peterlik, and K. Kromp, "A novel resonant beam technique to determine the elastic moduli in dependence on orientation and temperature up to $2000^{\circ} \mathrm{C}$," Review of Scientific Instruments, vol. 70, no. 7, pp. 3052-3058, 1999.

[11] K. Heritage, C. Frisby, and A. Wolfenden, "Impulse excitation technique for dynamic flexural measurements at moderate temperature," Review of Scientific Instruments, vol. 59, no. 6, pp. 973-974, 1988.

[12] R. M. Digilov, "Flexural vibration test of a cantilever beam with a force sensor: fast determination of Young's modulus," European Journal of Physics, vol. 29, no. 3, p. 589, 2008.

[13] American Society for Testing and Materials, "Standard test method for dynamic Young's modulus, Shear modulus, and poisson's ratio for advanced ceramics by impulse excitation of vibration," Standard C 1259-01, April 2001.

[14] Comité Européen de Normalisation, "Determination of dynamic elastic modulus by measuring the fundamental resonant frequency," Standard EN 14146, 2004.

[15] D. J. Inman, Engineering Vibration, Prentice-Hall, Englewood Cliffs, NJ, USA, 1994.

[16] S. S. Rao, Mechanical Vibrations, Addison-Wesley, Menlo Park, Calif, USA, 3rd edition, 1995.

[17] K. R. Chun, "Free vibration of a beam with one end springhinged and the other free," ASME Journal of Applied Mechanics, vol. 39, no. 4, pp. 1154-1155, 1972.

[18] "Multilab software for MultiLogPRO," Fourier System, http:// fourieredu.com/.

[19] Mathematica 7, Wolfram Research, Champaign, Ill, USA, 2008.

[20] J. R. Davis, Ed., Metals Handbook, ASM International, Materials Park, Ohio, USA, 1990. 

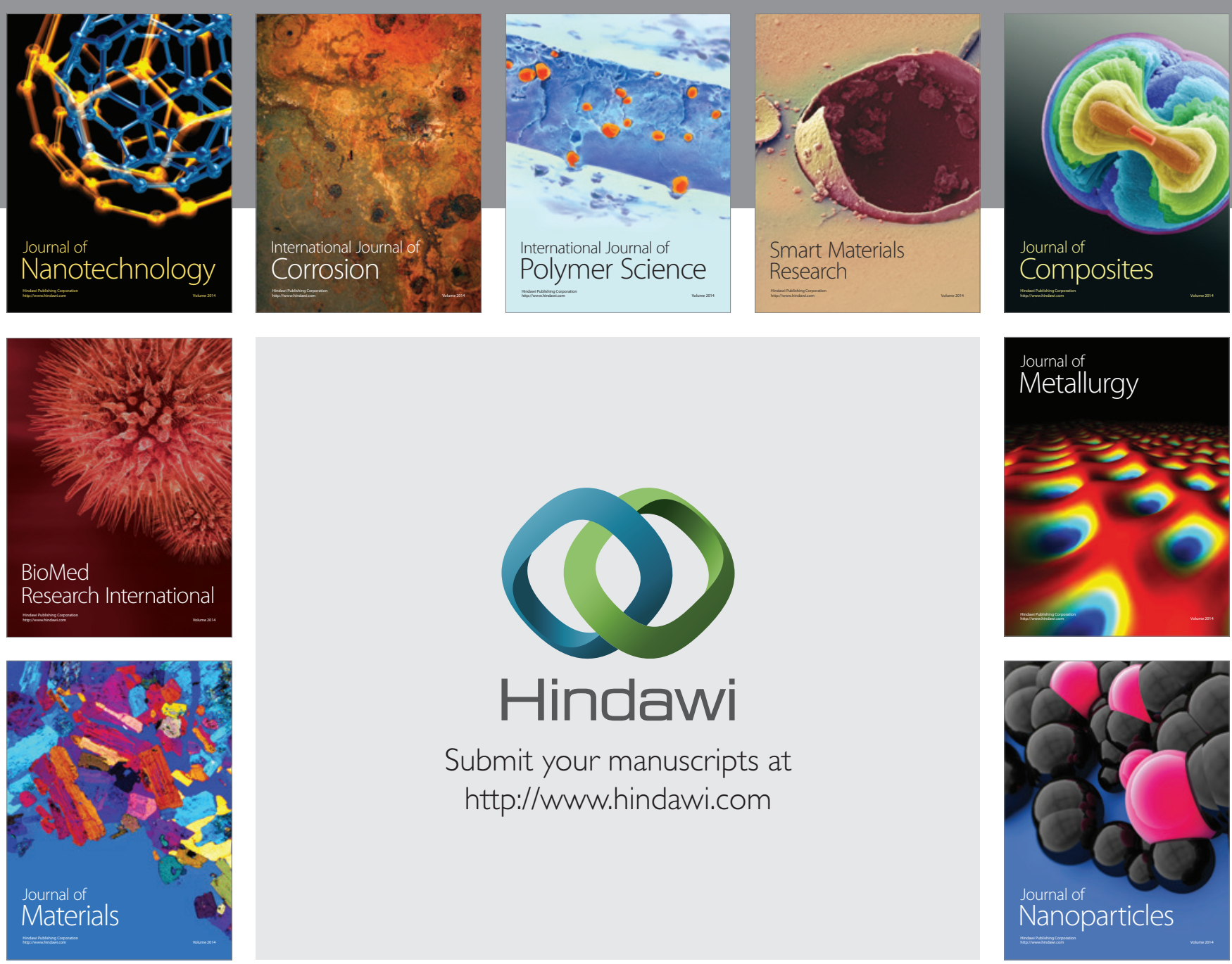

Submit your manuscripts at http://www.hindawi.com
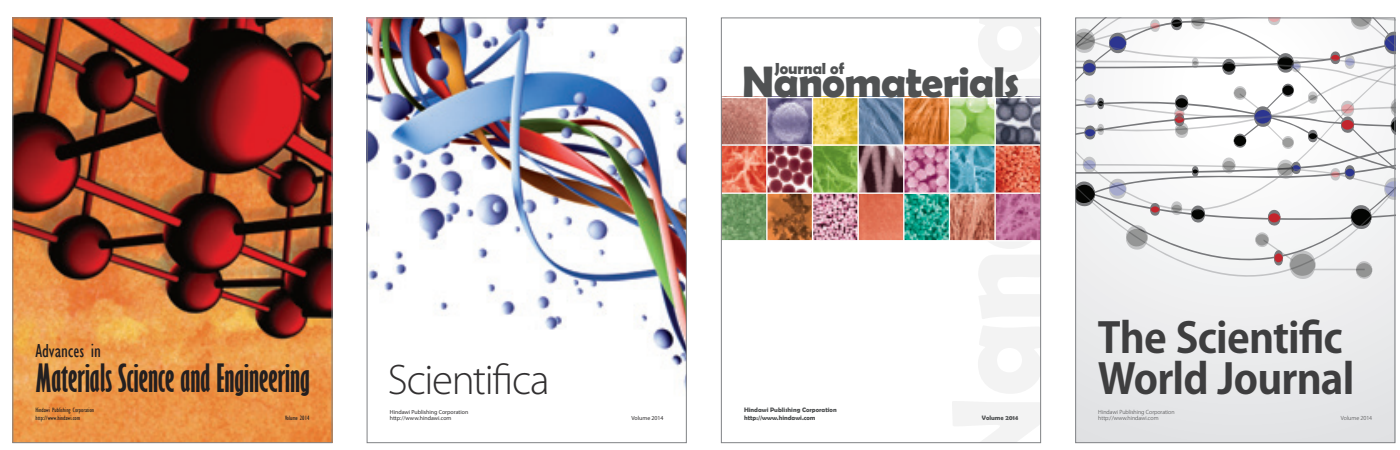

\section{The Scientific World Journal}
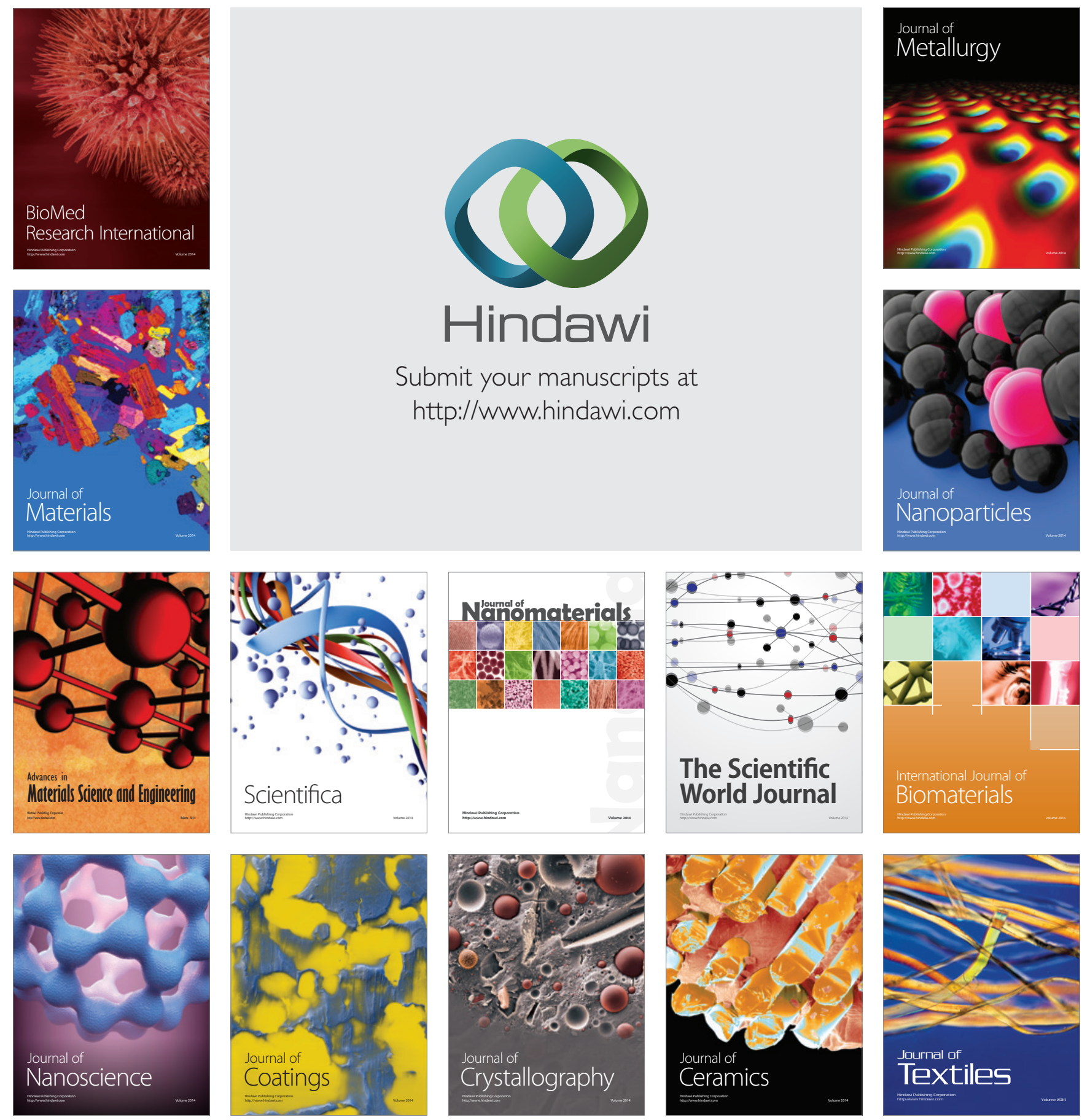